\title{
Ratios of record high to record low temperatures in Europe exhibit sharp increases since 2000 despite a slowdown in the rise of mean temperatures
}

\author{
Martin Beniston
}

Received: 28 July 2014 / Accepted: 7 January 2015 / Published online: 18 January 2015

(C) Springer Science+Business Media Dordrecht 2015

\begin{abstract}
A study has been undertaken to analyze the behavior of record high and low values of temperature since the early 1950s for 30 locations spread across Europe. When establishing the ratios of the number of record Tmax to record Tmin values in each year, it is seen that there is a sharp increase in these ratios in the most recent decade. This seems to be an apparent paradox in view of the slow-down in atmospheric temperatures that has been observed since the early 2000s at both the hemispheric and European scales, but closer analysis suggests that the relationship between the record high:low ratios and mean annual temperatures is not linear but rather a square relationship. It is suggested that the record high to record low ratios in both the Mediterranean region and beyond $60^{\circ}$ latitude north, observed in the most recent decade, may be related to an amplification of low-level atmospheric temperatures resulting from shorter snow seasons in the north and enhanced summer dryness in the south.
\end{abstract}

\section{Introduction}

There has been considerable media and scientific interest in the apparent hiatus in global warming over the past 10-15 years, with claims that there has been no significant warming since the end of the 1990s (e.g., Trenberth and Fasullo 2013; Pretis and Allen 2013; Held 2013). Some graphical evidence on the hemispheric and European scales will be provided later in Section 2. The slowdown in rising temperatures masks many other indicators that continue to exhibit increasing trends even in the past decade. Seneviratne et al. (2014) for example mention that "observational data show a continued increase of hot extremes over land during the so-called global warming hiatus. This tendency is greater for the most extreme events and thus more relevant for impacts than changes in global mean temperature". Indeed, in Europe alone, the decade 2001-2010 has seen some of the most extreme and persistent heat waves on record, in particular the devastating 2003 European summer (e.g., Beniston 2004; Schär et al.

Electronic supplementary material The online version of this article (doi:10.1007/s10584-015-1325-2) contains supplementary material, which is available to authorized users.

M. Beniston $(\bowtie)$

Institute for Environmental Sciences and Department of Physics, The University of Geneva, Geneva,

Switzerland

e-mail: martin.beniston@unige.ch 
2004), and the equally-dramatic summer of 2010 in western Russia (e.g., Barriopedro et al. 2011) and elsewhere (e.g., Orlowsky and Seneviratne 2011).

This paper will report upon other statistics that serve to highlight the fact that although the rise in annual temperatures since the early 2000s may be much slower than in the previous decade(s), signs of rapid change are still very much present. The starting point for the present study is based on a paper by Meehl et al. (2009), who investigated the evolution in the ratios of record high maximum temperatures (Tmax) to record low minimum temperatures (Tmin), and found for several thousand locations across the United States that the number of record highs are today roughly double the number of record lows, possibly rising to a ratio of 50:1 by 2100 .

The present study uses a somewhat different approach to that reported by the aforementioned paper. Exploiting data from 30 different locations spread across Europe since 1951, the ratios in the number of days per year with record high Tmax and record loe Tmin will be investigated for each calendar day for the 60 -year period. It will be demonstrated that the ratio of record highs to record lows has increased throughout Europe during this period, and particularly since the turn of the 21 st century. The paper will thus attempt to relate these statistics to the evolution of mean temperatures in the given timeframe, and identify possible underlying causes for the observed geographical distribution in these record high-to-low ratios and their trends.

\section{Data and methods}

Figure 1 presents the 30 European stations used in this study that have been selected on the basis of data availability and quality since 1951 through to end-2013. The climate data analyzed in this paper is made available European Climate Assessment and Data archive (ECA\&D; Klein Tank et al. 2002). ECA\&D data is updated on a monthly basis and qualitychecked for homogeneity, making it one of the more reliable observational data sets for Europe. Only homogenous data has been selected for this study, in order to ensure greater robustness in the extreme tails of the temperature PDFs that are sensitive to the quality of observational data.

Figure 2 suggests that for the Northern Hemisphere, the rising trend of temperatures that was particularly sustained from the 1980 s to the early 2000 s has tailed off in the past decade. This figure also plots the annual temperatures anomalies (computed on the basis of the 19611990 baseline) for the 30 European locations used in this study and defined in Fig. 1; the data has been smoothed with a 5-year running mean. The European records highlight the fact that multi-annual / decadal trend reversals have existed in the past, for example in the late 1950s or the mid-1970s, while nevertheless embedded within a clearly-rising trend of about $1{ }^{\circ} \mathrm{C}$ in the last 30 years of the record.

Unfortunately not all of the many stations integrated within ECA\&D span the duration required for this study (i.e., from 1951 to 2013), so that it was necessary to limit our study to the 30 stations that are mapped in Fig. 1. These data fulfill the requirements that were a prerequisite for the present paper, namely length of record; homogeneity of the data; and a representative geographical spread across the continent. For some of the stations, where data is missing for short periods, simple linear interpolation based on the neighboring data points has enabled to fill these gaps, with a very low risk of missing important low or high record temperatures.

In a similar manner to previous studies undertaken on European temperature modulation by dry conditions (Beniston 2012) and on "climate analogs" (Beniston 2013), a number of continent-wide transects have been defined. In the previous studies by this author, transects 


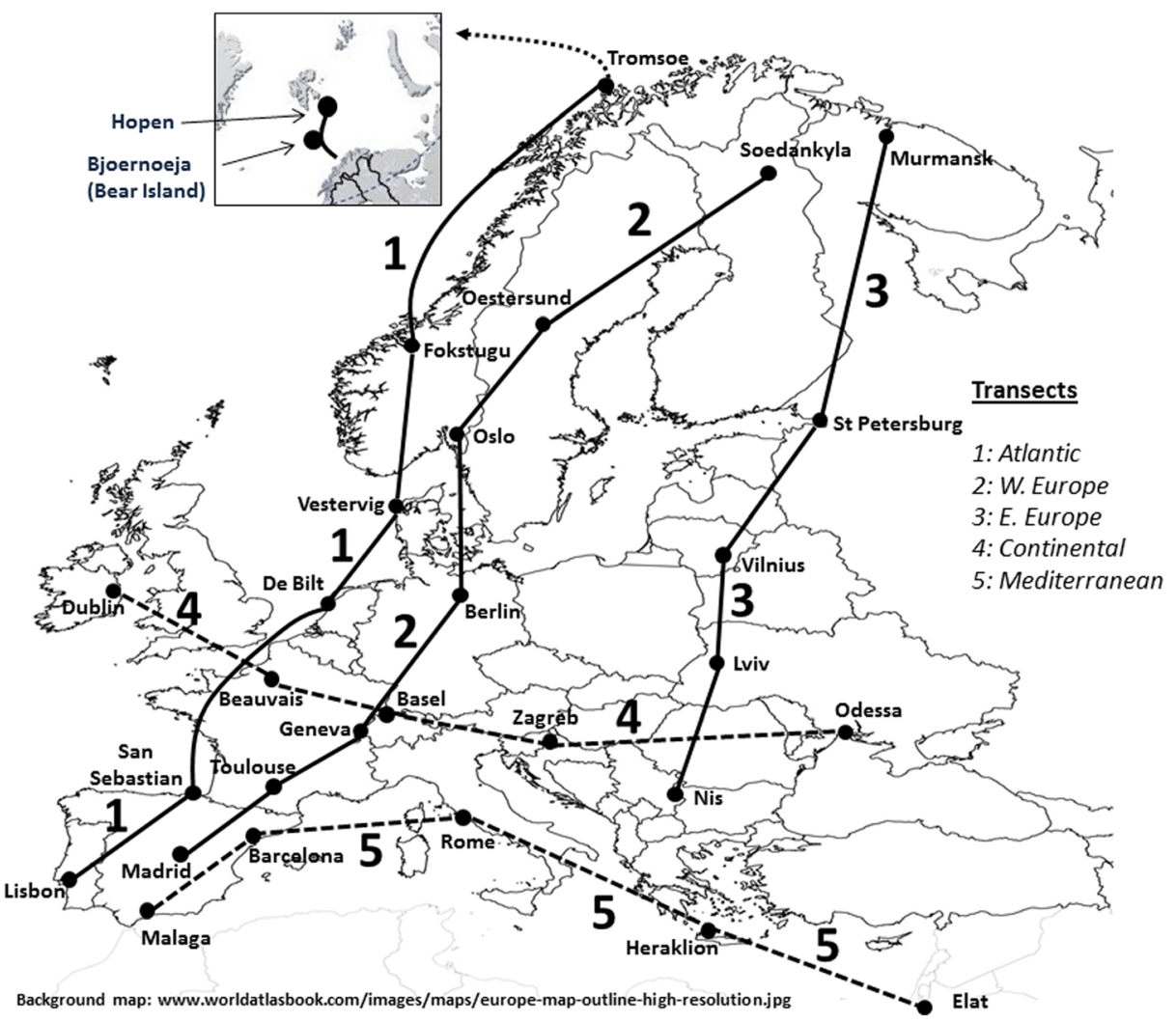

Fig. 1 Map of Europe illustrating the 30 climate observing stations used in this study, and the 5 transects that link these stations

were established either on the basis of their potentially-common characteristics (e.g., Mediterranean-type climates), or the latitudinal temperature gradients that may differ from or be similar to - one another according to where on the continent these transects have been located (e.g., Atlantic seaboard; Eastern Europe; etc.). For example, in Beniston (2012), the transect approach used there helped identify three regimes of dryness in Europe that enabled a clustering of stations with similar climatic characteristics. In the present study, the transects have been established to emphasize their potentially-common characteristics (e.g., Mediterranean-type climates), or to highlight possible latitudinal temperature gradients that may differ from - or be similar to - one another according to where on the continent these transects have been located (e.g., Atlantic seaboard; Eastern Europe; etc.). As will be shown in the paper, the transect approach enables a possible explanation of some of the findings related to extremes of heat or cold. The transects established for the purposes of the present paper enable a wide range of climates to be sampled along three north-south and two east-west continental sections, namely:

1. the Atlantic seaboard from Lisbon through to Hopen, located off the main island of Svalbard in the Arctic Ocean;

2. from Spain to Finland in the western half of Europe;

3. from Serbia to the Arctic Ocean in the eastern part of Europe; 
4. a cross-continental Atlantic-to-Black Sea transect; and

5. a Mediterranean segment from southern Spain to Elat, an Israeli port and resort on the Red Sea.

In order to use extreme values of heat or cold as an indicator of climatic trends, the high and low records over the 63 years 1951-2013 have been determined for each day of the year, i.e., by identifying the hottest and coldest Tmin and Tmax for each calendar day from January 1 through December 31. The procedure could be summarized in mathematical shorthand as:

$$
\min [\operatorname{Tmin}(\mathrm{D}, \mathrm{Y})] \text {; and } \max [\operatorname{Tmax}(\mathrm{D}, \mathrm{Y})]
$$

where Tmin and Tmax are the daily minimum and maximum temperature values, respectively, and $\mathrm{D}$ and $\mathrm{Y}$ are the day and year time parameters that are iterated over 365 calendar days for each of the 63 years from 1951 to 2013. In this way, over the selected measurement period, 365 values of Tmin and Tmax record high and record low values have been returned for each of the observational stations identified in Fig. 2. Then, for each year, the sum of Tmin and Tmax low and high records have been calculated whenever a given calendar day returns a particular record value. For example in Oslo and for the year 1995, a total of 2 record lows (i.e., the coldest values of Tmin for a 17th of May and an 8th of August during the 63 years of record) and 3 record high Tmax days (the warmest values of Tmax for a 24th of April, 22nd of August, and 19th of October) have been identified. Using this approach for all 30 European sites and for all years since 1951, the ratios between the number of record high Tmax and record low Tmin days per year, as well as the same statistics for Tmin and Tmax taken separately, have been computed.

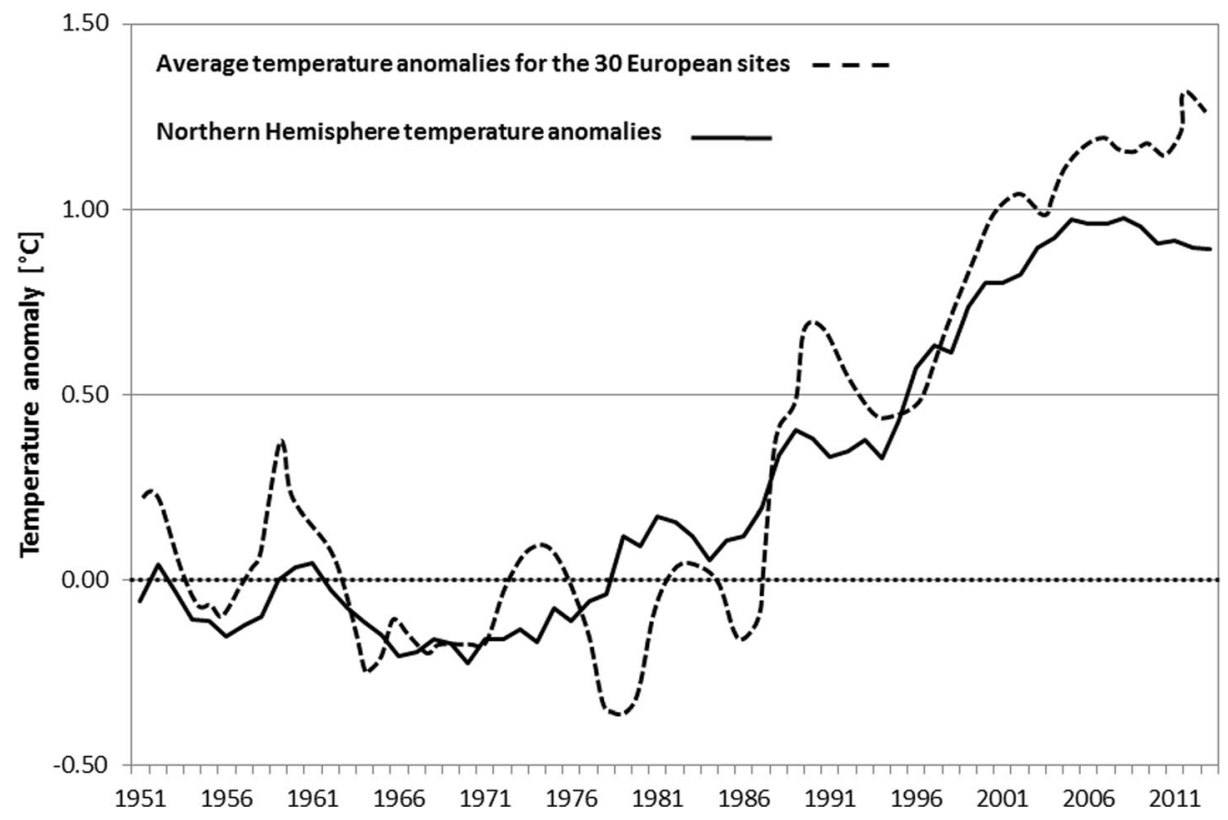

Fig. 2 Mean temperature anomalies of the 30 European stations defined in Fig. 1 are given by the dashed line. Northern Hemisphere anomalies are shown by the solid line, using data from the Climatic Research Unit, University of East Anglia, UK. Both the solid and dashed lines are 5-year running means 
There are of course numerous other metrics that can be used to define the behavior of distribution tails or test the significance of trends in such metrics. The metric used here may in some instances more readily understood by non-experts. For example, a statement such as "there are more than four times the number of record high temperatures than record low temperatures today than was the case 30 or 40 years ago" more readily conveys the notion of a significant change than more abstract notions of shifts and trends in the tails of probability density functions. In a world where Science often has difficulties in communicating efficiently with Policy, relatively simple metrics that are readily understood can become powerful tools to convey scientific outputs to various end-users (general public, policymakers, stakeholders, etc.). Indeed, it is for this reason that ratios of record high: record low values of temperature have been used here, because using differences as opposed to ratios will not necessarily communicate quite the same message. Furthermore, ratios can exhibit similar values, (i.e., twice of four times record highs over record lows...) in regions where the absolute numbers may be very different - and where the use of differences between high and low record numbers may mask what are indeed significant changes over time.

\section{Results and discussion}

Based on the analyses of the ratio of the number of days with record high and record low temperatures, two principal questions arise that this paper will attempt to answer, namely:

1) Why do the ratios of high to low records of Tmin and Tmax increase rapidly in the most recent decade of the record despite a slow-down in Northern Hemisphere and European temperature trends during this same decade?

2) Are there mechanisms that can serve to explain the geographical and temporal differences in high:low record temperature ratios?

\subsection{Time evolution of record high Tmax:record low Tmin ratios}

Repeating the procedure employed by Meehl et al. (2009), Fig. 3 shows the decline in the number of records of daily minimum (Tmin) and daily maximum (Tmax) temperatures. It is seen on the logarithmic scale that the decline scales with a $1 / \mathrm{n}$ profile, whose curve has been added to this figure; $\mathrm{n}$ is the number of data points for a given location (i.e., time in this instance, namely 365 days x 63 years). In the first year of a time series, daily temperatures are all obviously record values, but as time goes by, additional records are increasingly difficult to come by. Arnold et al. (1998), Meehl et al. (2009) or Rahmstorf and Coumou (2011) inter alia have demonstrated that in a stationary system, the number of records observed over time $n$ should decrease as $1 / n$. The likelihood of exceedance of a particular record is thus represented by an asymptotic curve with probability $1 / \mathrm{n}$.

Meehl et al. (2009) further show that deviations from the theoretical curve can be the result of different natural variability and/or long-term non-zero trends in temperature. The inset graph in Fig. 3 further shows the evolution over the period of record of the ratios in the number of records of Tmax over those of Tmin. By the end of the time series, Tmax/Tmin ratios rise to about 1.5 , although the graph is clearly very noisy. The ratio found here is somewhat lower than the factor of 2 observed by Meehl and co-workers - a possible consequence of the lower number of stations used here - but nevertheless confirms that in Europe too, there are similar shifts towards warmer records. 


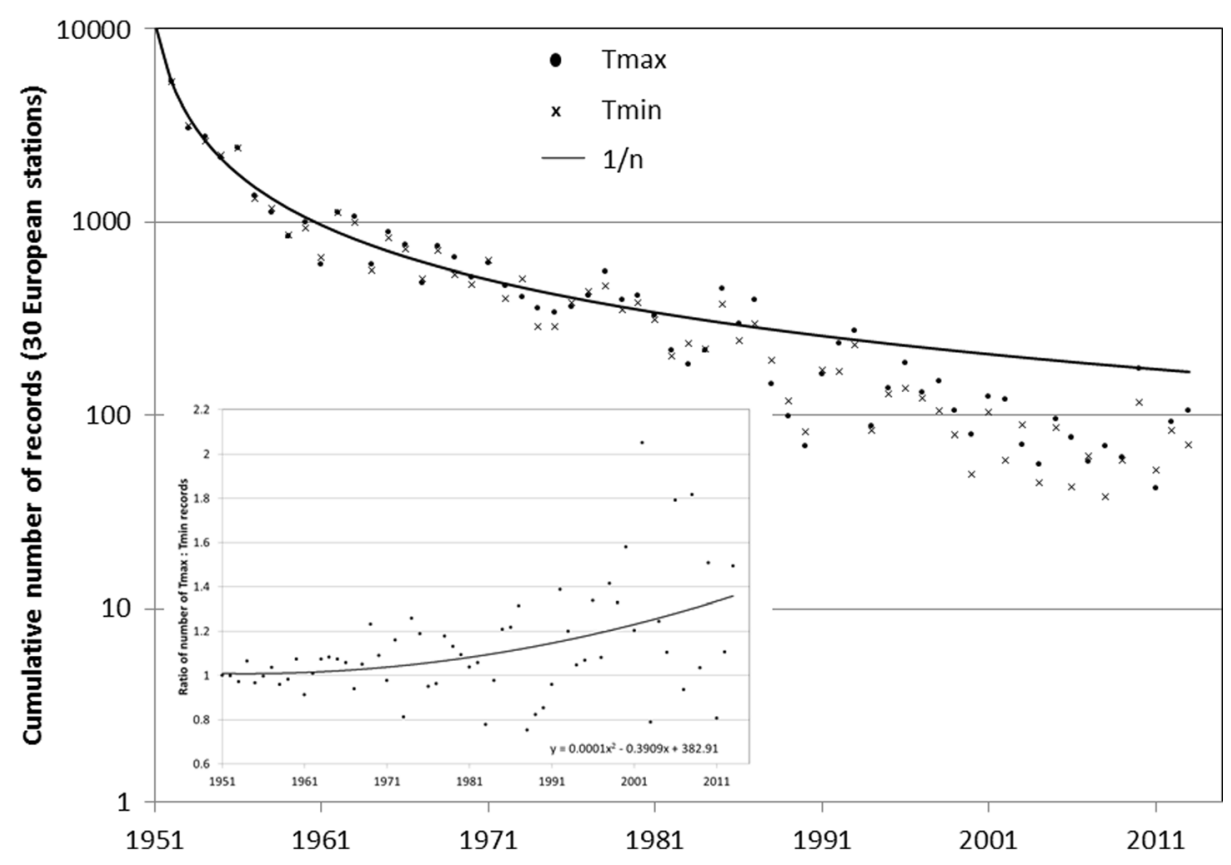

Fig. 3 Time series of the number of Tmin and Tmax records cumulated for the 30 European sites, following Meehl et al. (2009). Inset graph shows the evolution of the ratio of the number of records of Tmax vs Tmin, with the best-fit curve superimposed

Figure 4 provides an example of the course of record low Tmin and record high Tmax for Lisbon, Portugal, since 1951. 35 record values of Tmin were measured during the very cold winter of 1956, while 24 record high Tmax values occurred in 2010 (more than the intense 2003 European heat-wave summer, with 13 records). From the 1960s to the 1990s, there is an alternation of years with similar numbers of low and high records; the situation changes in 1987, which is the "crossover year" following which the record high values of Tmax largely outnumber the record low values of Tmin. In this paper, the crossover point is identified as the year beyond which there are no more than two events through to 2013 during which the number Tmin records exceed the number of Tmax records. This is of course a rather arbitrary choice, but is based on the fact that less than $5 \%$ of the data points beyond the crossover year exhibits an event where the number of days with Tmin records outnumber those with Tmax records.

The inset graph in Fig. 4 highlight the linear trends in the number of days per decade for the entire 60-year record. For Lisbon, the sharpest decline in the number of Tmin records occurs in the 1970s (roughly 1 day per year), and the trend remains negative thereafter (but very close to zero in the 2000s because the number of minimum temperature records fluctuates within 0 to 1 days per year). In contrast the linear decadal trends of Tmax record numbers fluctuates around the zero line until the most recent decade, where a strong trend of almost 12 days/ decade is observed.

In the case of Bear Island (located between the North Cape of Norway, and Svalbard at $74.5^{\circ}$ latitude north, not shown), the $1960 \mathrm{~s}$ were characterized by a particularly large number of record low values of Tmin (32 in 1962 and 44 in 1968) and a small number of record high values of Tmax (between 0 and 5 during the 1960s). In the first decade of the 21 st century, 


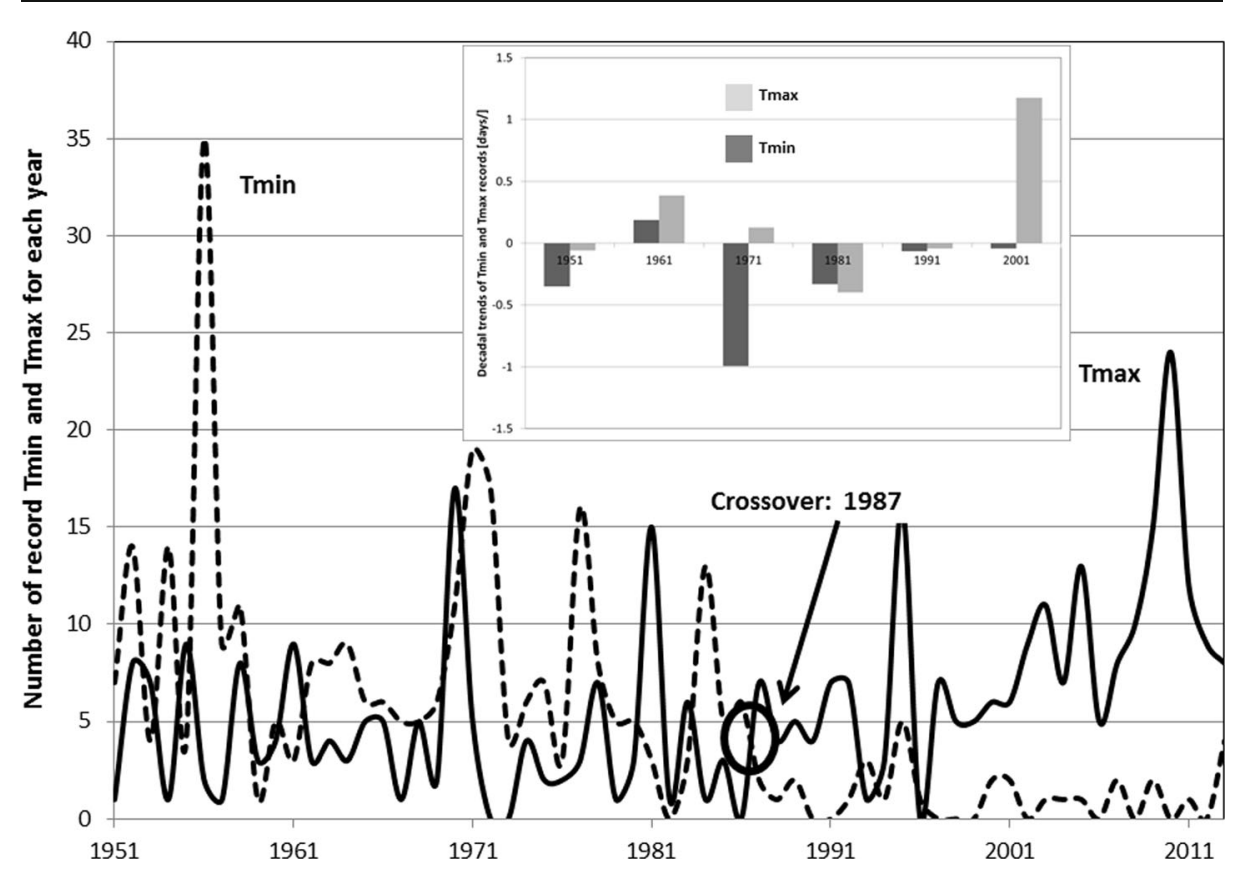

Fig. 4 Example of the evolution of the number of Tmin and Tmax records for Lisbon, Portugal, for each year between 1951 and 2013. The inset graph shows the linear trend in the number of record days per decade for the entire 60-year record

several years exhibit up to 30 record high values, as in 2006, while the number of record lows ranges from 0 ( 5 years in a row from 2006 to 2010) to 2 . The crossover year for Bear Island as defined above, beyond which Tmax record begin to outnumber Tmin record, is 1995.

Figure 5 illustrates the decadal-scale ratios of numbers of high Tmax:low Tmin records for all stations in the 5 European transects defined in Fig. 2. The use of aggregated data for each decade does not enable robust statistical trend analyses, but the choice has been made here for the purposes of clarity compared to the noisiness of annually-resolved data. In addition, data aggregated over several years avoids problems of zero values that can occur in individual years.

For almost all transects, the ratios are seen to range generally from between 0.5:1 and 2:1 up to the 1980 s, but in the 1990 s and particularly in the first decade of the 21 st century, there is a very sharp rise in their values. Along Transect 1, and in the decade 2001-2010, ratios span a range from 2.6:1 in San Sebastian, Spain, to 25:1 in Hopen, Norway; from 3:1 in Madrid to close to 7:1 in Finland along Transect 2; from 3:1 to almost 8:1 on the eastern European segment (Transect 3) from Nis, Serbia, to St Petersburg and Murmansk, Russia. For the two east-west segments, ratios range from less than 3:1 in Dublin to close to 6:1 in Odessa (Transect 4), and in the Mediterranean sector (Transect 5) lie between 5:1 and 6:1.

In order to extend the analysis to earlier periods than the 1950s discussed here, a number of Swiss stations whose daily data is available at least the beginning of the 20th century have been analyzed. Figure 6 shows the decadal evolution of the number of high Tmax : low Tmin records since 1901 in Basel. This example helps highlight the fact that extending back to the beginning of the 20th century leads to identical conclusions as to the strong rise in ratios in the most recent decade. 
a

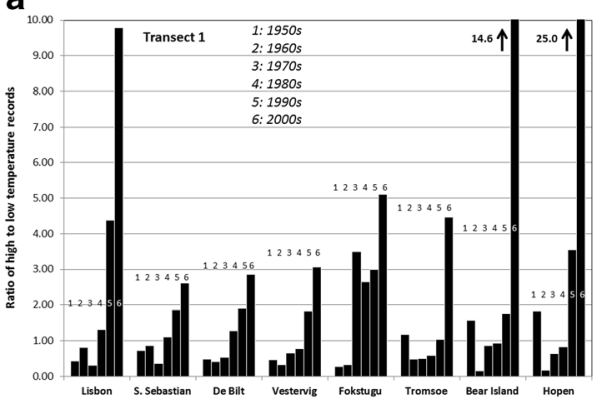

C

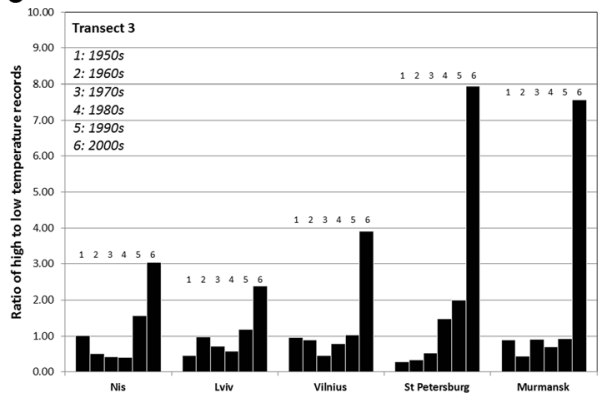

e

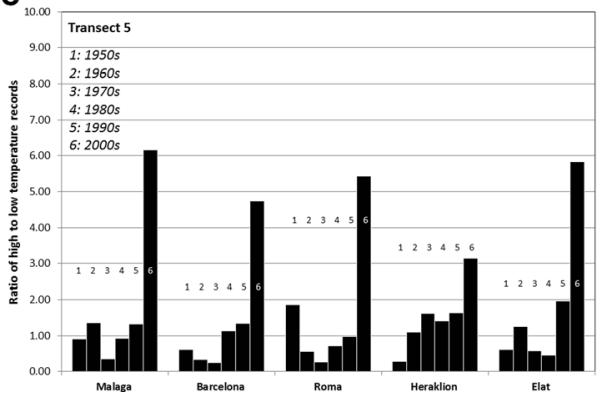

b

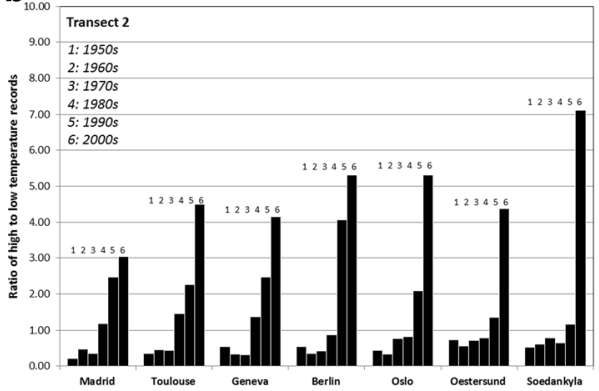

d

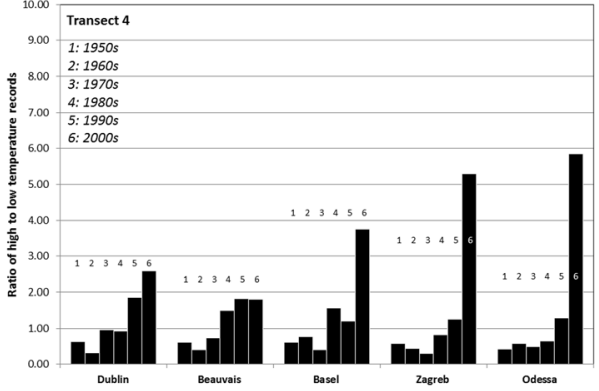

Fig. 5 a-e: Decadal-scale ratios of the number of Tmax to Tmin record temperatures along the 5 transects defined in Fig. 2

Conducting the same analyses that lead to the establishment of the numbers of record high Tmax to record low Tmin, Fig. 7 illustrates that if the upper and lower records of Tmin and Tmax are taken separately, the conclusions remain the same. In the example given here, for Barcelona, Spain, the ratios of numbers of record high:low Tmin and of Tmax events exhibit the same sharp rise in the most recent decade.

As previously mentioned there would be no statistical reason for hot records to be more frequent than cold records in a stationary climate, which is clearly not the case when analyzing the results portrayed in Figs. 4 and 5. A shift in the position of the mean of the probability density functions (PDF) of Tmin and Tmax towards warmer conditions is generally accompanied by a shift of the entire PDF towards warmer temperatures. If the change in variance is small, this implies that the tails of the distribution - where the record high and record low values are located - favor an increasing number of upper extremes at the expense of lower extremes. Even with a change in variance, where the distance between the tails expands, there 


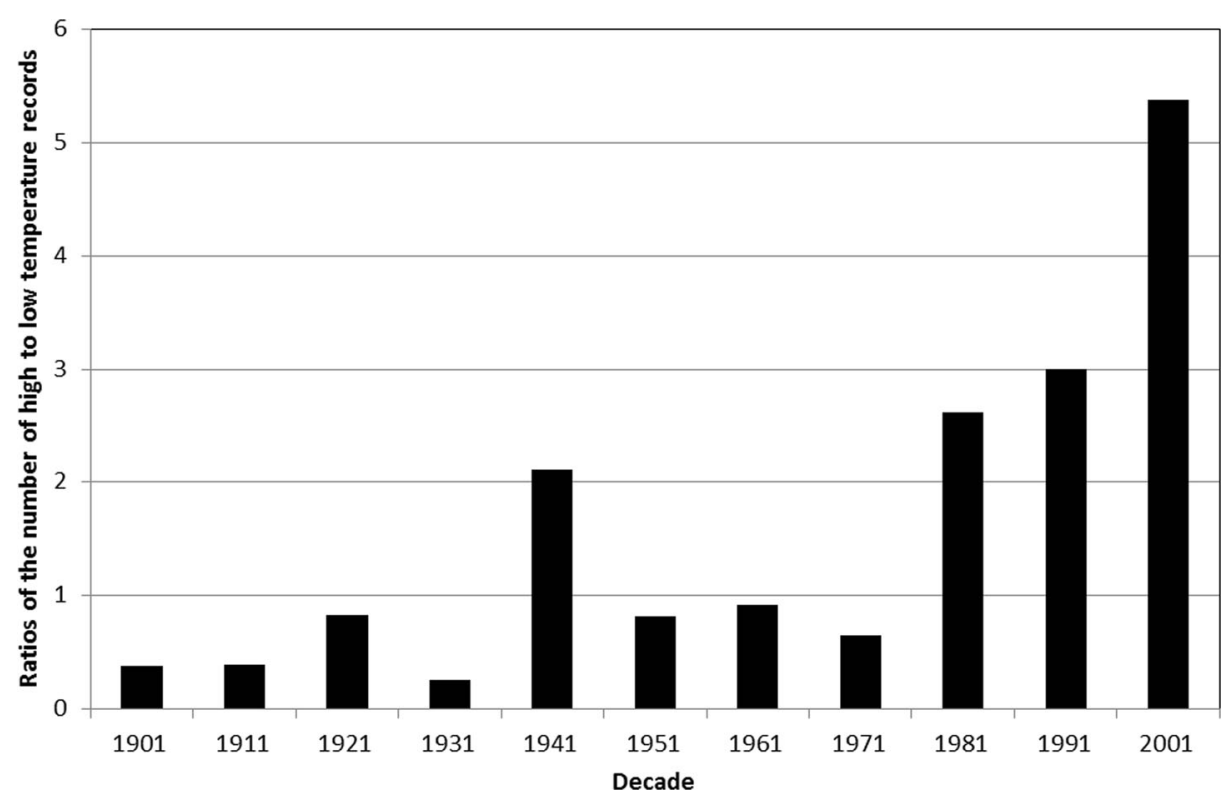

Fig. 6 Decadal-scale statistics of the number of Tmax : Tmin record days in Basel, Switzerland, to emphasize the fact that whatever the duration of the record, the latest decade exhibits the highest ratio values

is a strong likelihood of a disproportionate increase in the number of record high extremes compared to record low values. In general, it is seen that the tails of narrower distributions are highly sensitive to shifts in the mean; indeed narrow distributions are more a characteristic of the PDFs of Tmin than those for Tmax.

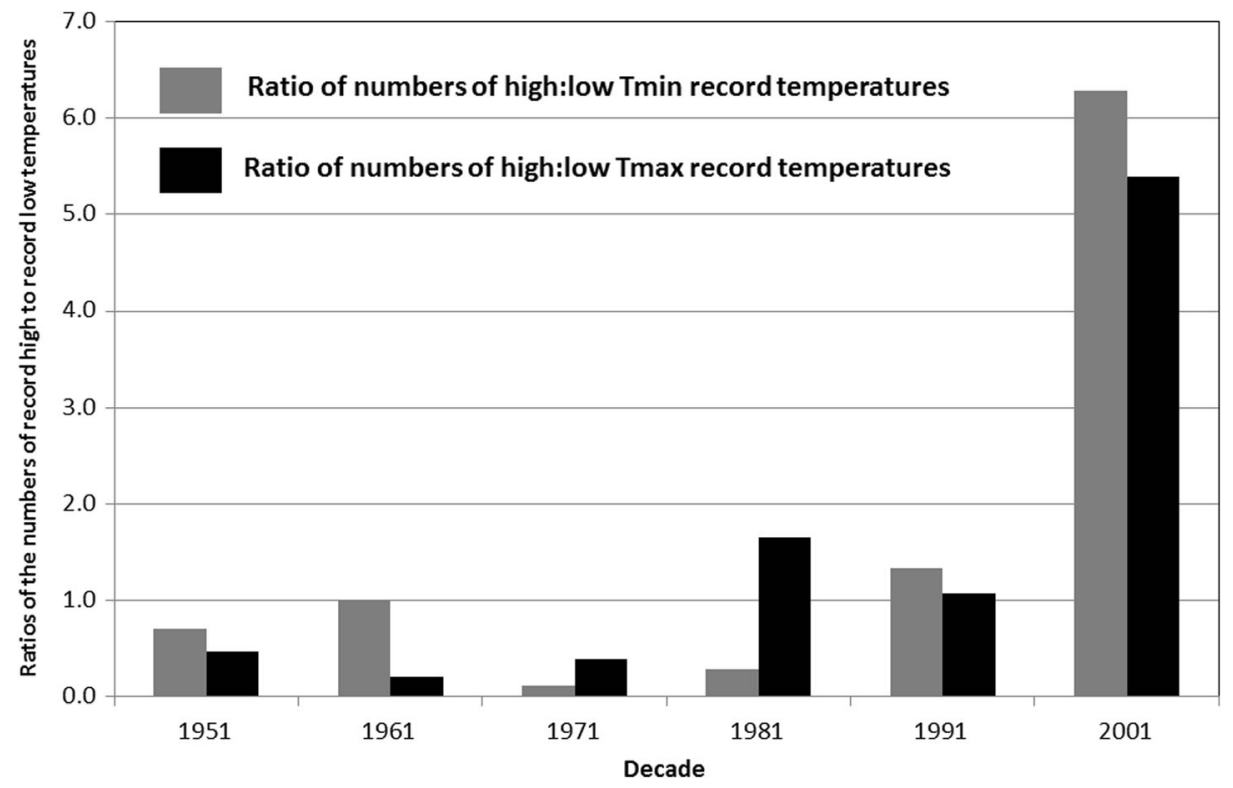

Fig. 7 Ratios of the number of upper : lower extremes of Tmin and Tmax taken separately 
The question remains as to why sharp increases in the value of high:low record temperatures are observed in the most recent part of the study period 1951-2013 without a correspondingly strong rise in temperatures since the end of the 1990s. It would seem intuitively reasonable to think that shifts in ratios could follow the rate of change in mean temperatures, i.e., strong increases in the number of Tmax records at the expense of Tmin records in the 1990 s, where the strongest rates of mean warming are observed, rather in the 2000s when rates of warming are a factor 2 or 3 lower. This apparent paradox can be at least partly be explained by examining the relationship between the mean temperature anomalies for all 30 stations and the high:low record days, provided in Fig. 8. Here, the average decadal temperature departures from their 1961-1990 climatological means, given on the abscissa, have been plotted against decadally-aggregated ratios on the ordinate. There are thus 180 data points (30 stations x 6 decades) that combine both the geographical spread of stations and their changes over time since the 1950s.

The best fit for the temperature anomaly to record ratios is defined by a quadratic curve, which helps explain close to $85 \%$ of the variance of the data plotted in this figure. Since the temperature-record ratio relationship is not linear, this can explain the strong increase in high:low record ratios between the 1990s and the 2000s despite the small additional rise in temperatures in the intervening period. The quadratic curve suggests that for "normal" temperature conditions, i.e., with a temperature anomaly of 0 , the record high:record low ratio range from 0.5:1 to 1:1 (i.e., close to parity between the number of days exhibiting record high Tmax and record low Tmin values in "normal" years), rising to $3: 1$ for a $1{ }^{\circ} \mathrm{C}$ anomaly and close to $9: 1$ for a $2{ }^{\circ} \mathrm{C}$ anomaly. Clearly, in the context of already high values of temperatures in the 1990s, any additional incremental warming - even apparently modest as seen in Fig. 1 can lead to strong increases in the record high:low ratios. Although the power relationship is modulated by a number of outliers in the upper-right segment of the graph, removal of these outliers (all ratio values above 7) still results in a quadratic curve that explains over $70 \%$ of the

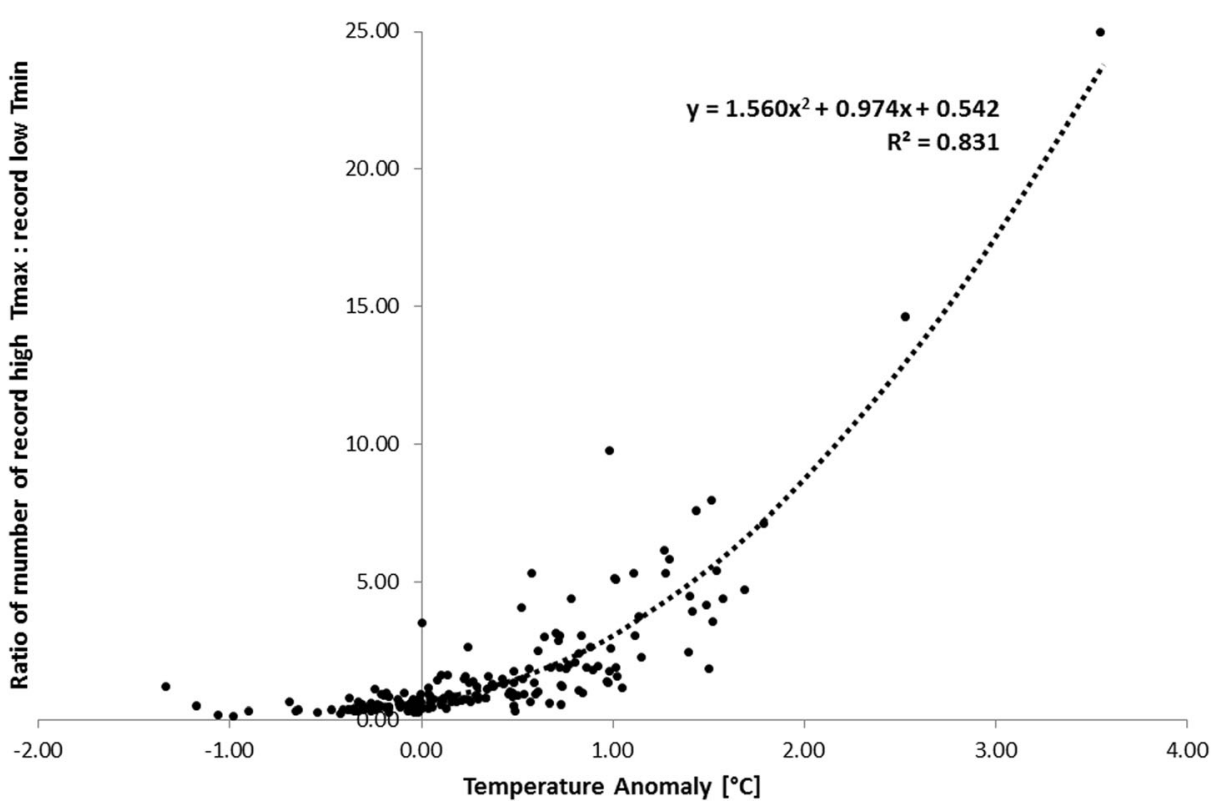

Fig. 8 Relationship between mean temperature anomalies and Tmax:Tmin record temperature ratios 
variance in the data. The statistical significance of the regression line is high at the $99 \%$ level for both the full curve and the curve without the outliers.

The non-linear relationship between a shift in mean temperatures and the number of record ratios can be further explained by shifts in the properties of the probability density functions (PDF) of the Tmin and Tmax distributions. As a result of changes in the position of the mean, a shift in the entire PDF is accompanied by an exponential rate of change in the tails of the temperature distribution (negative for Tmin; positive for Tmax), such that the ratios between the upper and lower tails may rapidly change with an apparently minor increase in mean temperature, as shown inter alia by Rahmstorf and Coumou (2011) and Simolo et al. (2011). In this latter paper, the authors comment on the fact that changes in the means of temperature are the main drivers of extreme heat in Europe, much more than shifts in variance or skewness of the PDF of Tmax. Additional work by Simolo et al. (2012) have shown that "The inherent nonlinearity between changes in PDF moments and tails becomes apparent ..., since the time evolution of EPs is controlled by the behavior of the underlying density function. For normal distributed data, for instance, rates of change in EPs [Exceedance Probabilities] scale exponentially in time".

As an example, the observational station of Lisbon, Portugal - that exhibits a strong change in the ratio of the number of Tmax:Tmin extremes - has been chosen to illustrate this point. Figure 9 shows the normal distributions of Tmin and Tmax, based on the mean and standard deviation properties of temperatures, for the 1950 s and the 2000s (i.e., the coldest and warmest decades of the series, respectively).

The warming in both series over the half-century $\left(1.5^{\circ} \mathrm{C}\right.$ for Tmin; $1{ }^{\circ} \mathrm{C}$ for Tmax $)$ sees a shift to the right of the PDFs of both Tmin and Tmax. Figure 10 highlights the implication of the overall shift in PDF on the speed of change in the respective lower Tmin and upper Tmax tails between these two decades. This figure simply shows the change, as a function of the observed temperatures in Lisbon, Portugal (Tmax above the zero line, Tmin below), in the

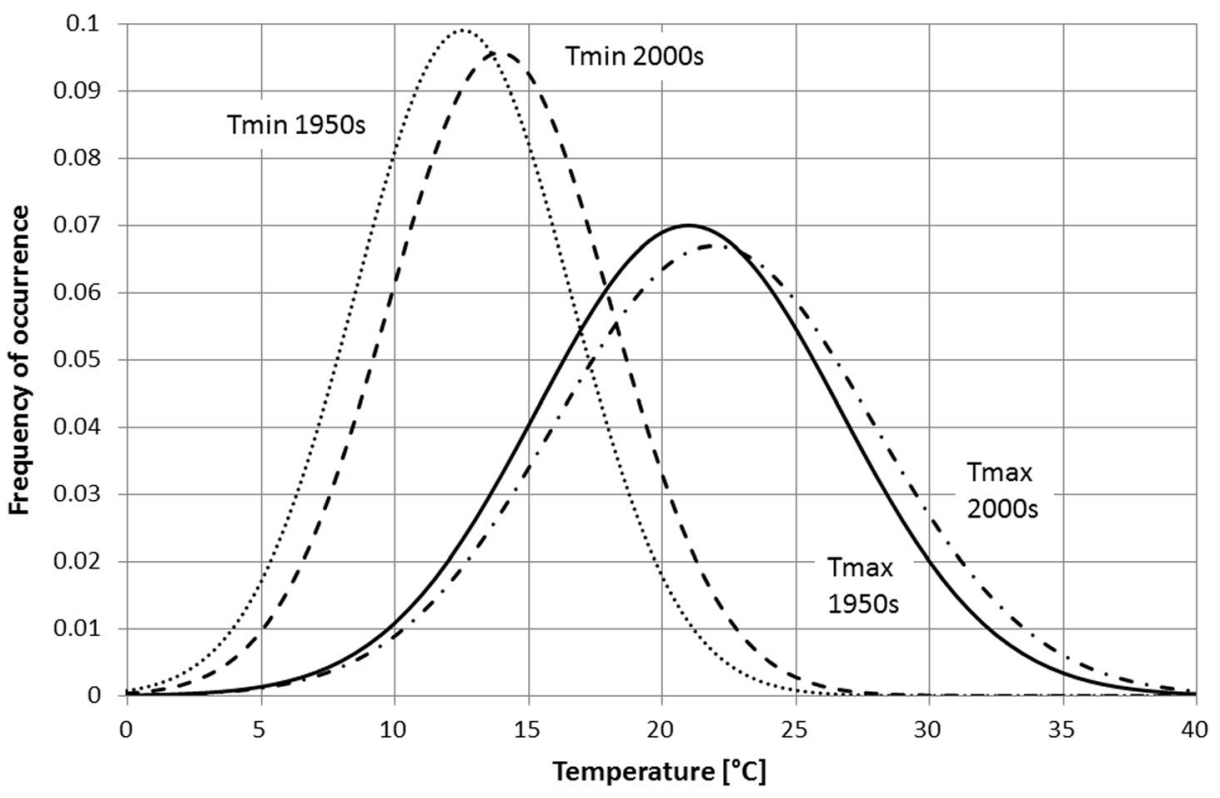

Fig. 9 Normalized probability density functions for Tmin and Tmax in Lisbon, Portugal, for the decade of the 1950 s and that of the 2000 s 


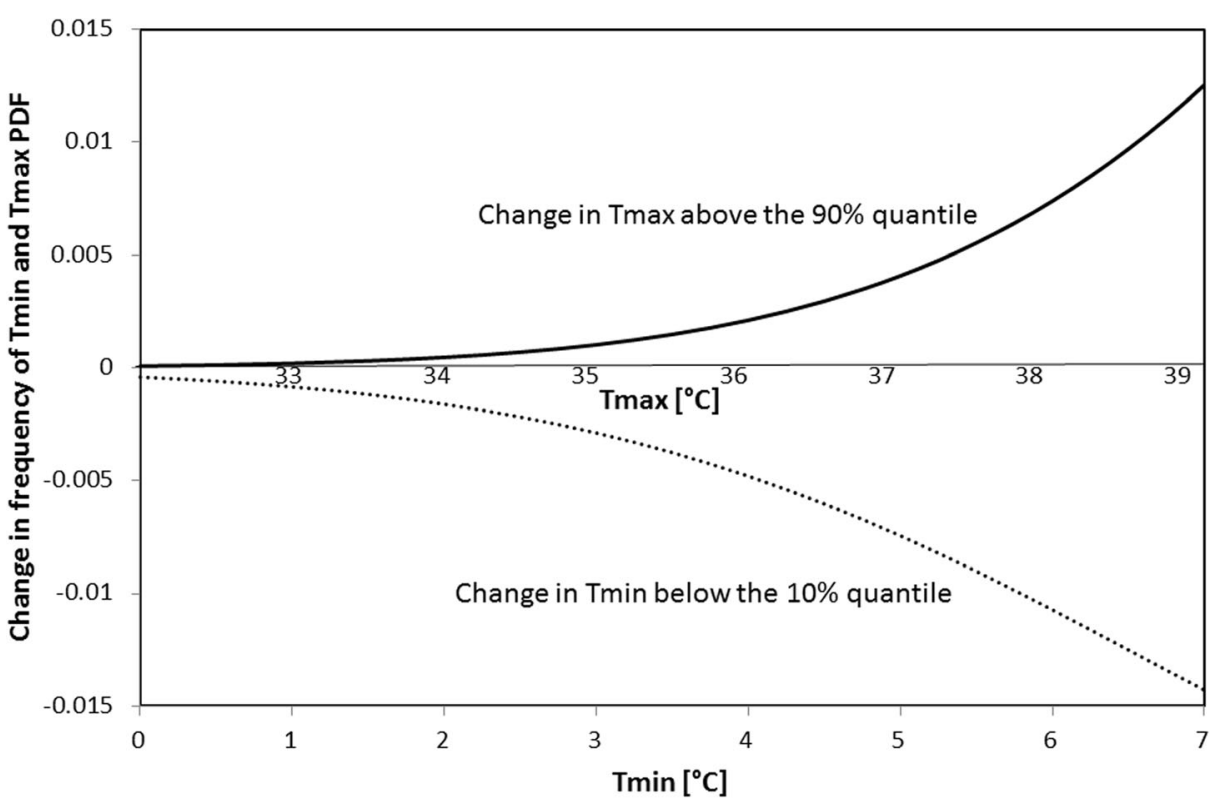

Fig. 10 Variation of the lower and upper tails of Tmin and Tmax, respectively, as a function of mean temperature (data for Lisbon, Portugal)

frequency of occurrence of Tmin below the $10 \%$ quantile and of Tmax above the $90 \%$ quantile, based on the normal cumulative distribution of temperature for that particular location. The decrease in frequencies of the lower tails of Tmin and the increase in frequencies of the upper tails of Tmax take on an exponential character that lead to the rapid divergence in the Tmin and Tmax frequencies with increasing temperatures, it is therefore not entirely surprising that very large ratios in the number of Tmax records over the number of Tmin records emerge in the most recent decade of the observational data.

\subsection{Geographical distribution of record high Tmax:record low Tmin ratios}

In the Supplementary Material available on-line, some ideas concerning the possible physical mechanisms that could explain the very high ratios, particularly for the Mediterranean zone and Northern Europe and the Arctic, are discussed. It is shown that shorter snow seasons in the northern latitudes and drier conditions in the Mediterranean domain may lead to positive temperature feedbacks and thus to a sharp rise in the number of Tmax records and decline of Tmin records.

\section{Concluding remarks and outlook}

The present study has shown that there has been a clear and massive increase in the number of days per year with record high Tmax: record low Tmin ratios in the most recent decade of the 1951-2013 temperature record for 30 selected observation stations in Europe. This sharp increase is seen to occur despite a reduction in the rate of warming since the early $2000 \mathrm{~s}$, as observed not only in Europe but also at the hemispheric scale. The sharp rise of high:low temperature ratios since 2000 , despite relatively small increases in mean temperatures in the 
last 10-15 years of the observational record, can be explained by a non-linear (quadratic) relation between mean temperatures and the Tmax:Tmin record ratios. It is suggested here that the increases may well be a consequence of increasing dryness during the summer in the Mediterranean region (where today there are on average 30 more dry days than in the 1950s), and a reduction in the cold season conducive to snow days in Arctic summers and Northern European springs (with up to 40 freezing days less than 60 years previously). Both effects can amplify positive temperature feedbacks in the lower atmosphere that in turn can lead to in strong increases in the number of record high Tmax values and correspondingly strong reductions in the number of Tmin records.

Future work could aim to use regional climate model projections to establish how these ratios may change in the future in a warmer world, i.e., to see whether the observed trends of high:low temperature records in the last $60+$ years would pursue their acceleration through to 2050 or 2100 , as suggested by the power curve exhibited in Fig. 8. Based on this curve, it is estimated that a sustained warming of $4{ }^{\circ} \mathrm{C}$ would lead to ratios close to $30: 1$, while a $5{ }^{\circ} \mathrm{C}$ warming in Europe would result in ratios of about 45:1. These figures are consistent with the findings reported by Meehl et al. (2009) for a simulated future climate.

\section{References}

Arnold BC, Balakrishnan N, Nagaraja HN (1998) Records. Wiley, New York, p 312

Barriopedro D, Fischer EM, Luterbacher J, Trigo RM, Garcia-Herrera R (2011) The hot summer of 2010: redrawing the temperature record map of Europe. Science 332:220-224

Beniston M (2004) The 2003 heat wave in Europe. A shape of things to come? Geophys Res Lett 31, L02022

Beniston M (2012) Exploring the behavior of atmospheric temperatures under dry conditions in Europe: evolution since the mid-20th century and projections for the end of the 21 st century. Int $\mathrm{J}$ Climatol. doi: $10.1002 /$ joc. 3436

Beniston M (2013) European isotherms move northwards by up to $15 \mathrm{~km} / \mathrm{year}$ : using climate analogues for awareness-raising. Int J Climatol. doi:10.1002/joc.3804

Held IM (2013) Climate science: the cause of the pause. Nature 501:318-319

Klein Tank A et al (2002) Daily dataset of 20th-century surface air temperature and precipitation series for the European climate assessment. Int J Climatol 22:1441-1453

Meehl, G.A., Tebaldi, C., Walton, G., Easterling, D., and McDaniel, L., 2009: Relative increase of record high maximum temperatures compared to record low minimum temperatures in the U.S. Geophysical Research Letters, 35, DOI: 10.1029/2009GL040736

Orlowsky B, Seneviratne SI (2011) Global changes in extreme events: regional and seasonal dimension. Clim Chang. doi:10.1007/s10584-011-0122-9

Pretis F, Allen M (2013) Climate science: breaks in trends. Nat Geosci 6:992-993

Rahmstorf S, Coumou D (2011) Increase of extreme events in a warming world. Proc Natl Acad Sci 108:1790517909

Schär C, Vidale PL, Lüthi D, Frei C, Häberli C, Liniger M, Appenzeller C (2004) The role of increasing temperature variability in European summer heatwaves. Nature 427:332-336

Seneviratne SI, Donat MG, Mueller B, Alexander LV (2014) No pause in the increase of hot temperature extremes. Nat Clim Chance 4:161-163

Simolo C, Brunetti M, Maugeri M, Nanni T (2011) Evolution of extreme temperatures in a warming climate. Geophys Res Lett 38, L16701. doi:10.1029/2011GRL048437

Simolo C, Brunetti M, Maugeri M, Nanni T (2012) Extreme summer temperatures in Western Europe. Adv Sci Res 8:5-9

Trenberth KE, Fasullo JT (2013) An apparent hiatus in global warming? Earth's Future 1:19-32 